\section{A Computer-Aided Method to Expedite the Evaluation of Prognosis for Childhood Acute Lymphoblastic Leukemia}

www.tcrt.org

This study presented a fully-automated computer-aided method (scheme) to detect metaphase chromosomes depicted on microscopic digital images, count the total number of chromosomes in each metaphase cell, compute the DNA index, and correlate the results to the prognosis of childhood acute lymphoblastic leukemia (ALL). The computer scheme first uses image filtering, threshold, and labeling algorithms to segment and count the number of the suspicious "chromosome," and then computes a feature vector for each "detected chromosome." Based on these features, a knowledge-based classifier is used to eliminate those "non-chromosome" objects (i.e., inter-phase cells, stain debris, and other kinds of background noises). Due to the possible overlap of the chromosomes, a classification criterion was used to identify the overlapped chromosomes and adjust the initially counted number of the total chromosomes in each image. In this preliminary study with 60 testing images (depicting metaphase chromosome cells) acquired from three pediatric patients, the computer scheme generated results matched with the diagnostic results provided by the clinical cytogeneticists. The results demonstrated the feasibility or potential of using a computerized method to replace the tedious and the reader-dependent diagnostic methods commonly used in genetic laboratories to date.

Key words: Acute Lymphoblastic Leukemia (ALL); Metaphase chromosomes; Chromosome analysis; and Evaluation of prognosis.

\section{Introduction}

Childhood acute lymphoblastic leukemia (ALL) is a disease in which too many underdeveloped infection-fighting white blood cells - lymphocytes - are found in children's bone marrow and blood. Those leukemia cells can replace normal blood cells and spread to other important organs such as liver, spleen, kidney, and the central nervous system. ALL is the most common cancer in children and is among one of the most curable cancers in pediatric malignancies (1). ALL accounts for $75 \%$ of all cases of childhood leukemia, with nearly 3,000 children in the United States and 5,000 children in Europe diagnosed with ALL every year (2). The outcome for children with ALL has improved dramatically with the development and progress of therapeutic methods. The survival rate for children under 15 years old reaches $75 \%$. Despite this great improvement, there are still $25 \%$ of pediatric patients who suffer from ALL, fail to response to the regular therapy, and experience a cancer recurrence. Relapsed leukemia is the third most common childhood cancer. On the other hand, some leukemia patients are treated too heavily even if they are highly curable with a small dosage or less chemical treatment. This can cause serious side effects to the patients (3). Thus,
Xingwei Wang, MS

Shibo Li, M.D. ${ }^{3}$

Hong Liu, Ph.D. 1,*

John J. Mulvihill, M.D. ${ }^{3}$

Wei Chen, Ph.D. ${ }^{4}$

Bin Zheng, Ph.D. ${ }^{2}$

${ }^{1}$ Center for Bioengineering and School of

Electrical and Computer Engineering

University of Oklahoma

Oklahoma, USA

${ }^{2}$ Department of Radiology

University of Pittsburgh Medical Center

Pittsburgh, PA, USA

${ }^{3}$ Department of Pediatrics

University of Oklahoma Medical Center

Oklahoma City, OK, USA

${ }^{4}$ Department of Physics and Engineering

University of Central Oklahoma

Edmond, OK, USA
"Corresponding Author:

Hong Liu, Ph.D.

Email: liu@ou.edu 
a major challenge for the effective treatment of children with ALL is to find better methods to evaluate each case and predict its prognosis (good or poor). This may be important to help physicians select optimal therapeutic methods and, hence, cure more pediatric patients.

A number of clinical, genetic, molecular features have been identified and investigated to correlate or predict the outcome of patients with ALL (1). Among them, age, WBC (the number of white blood cell), and sex are the standard clinical time-honored features used to determine the intensity of treatment. DI (DNA Index - the ratio of DNA content of leukemia cells to the normal human diploid cells or nuclei) and the number of chromosomes (Ploidy) as genetic features are the prognosis factors used to predict the outcome of pediatric patients' treatment. Although chromosome band patterns are considered one of the most important and sensitive features in diagnosis of variety of cancers, ALL can be reliably classified into four categories based on the modal number of chromosomes depicted on identified metaphase cells (2). These four categories are: (i) hyperdiploid defined by more than 47 chromosomes with DI greater than 1.0 (35-45\% of cases); (ii) pseudodiploid defined when DI is equal to 1.0 and the number of chromosomes with structural or numeric abnormalities is 46 (About $40 \%$ of cases); (iii) diploid (46 chromosomes, DI of 1.0, about $8 \%$ of cases); and (iv) hypodiploid (fewer than 46 chromosomes, about $8 \%$ of cases; DI less than 1.0). DI is a statistically significant indicator and the strongest prognosis factor to evaluate the treatment outcome when compared to age, WBC, and sex in a POG (Pediatric Oncology Group) study (4). In general, patients with ALL having a DI between 1.16 and 1.6 have been reported to have a better prognosis than those with a DI less than $1.16(5,6)$. Specifically, "high" hyperdiploidy (defined as the number of chromosomes larger than 50 or by a DNA Index [DI >1.16 (7-9)] measured by flow cytometry (10-13), has very favorable prognosis. On the other hand, pseudodiploidy or hypodiploidy (With DI <1.16), and lack of common ALL antigen (CALLA) expression are typically associated with a poorer treatment outcome $(14,15)$.

In order to acquire and analyze those genetic features, as well as predict prognosis or predict the efficacy of the patients' treatment, technologists in a genetic lab need to first prepare a number of testing slides (usually 4-10) for each patient (16). Chromosome gain or loss due to preparation method is an important issue in affecting diagnostic accuracy of leukemia. Hence, technologists in genetic laboratory need to recognize whether a metaphase cell is incomplete or over complete due to preparation bias. As a common testing practice, technologists usually choose approximately 20 of the analyzable metaphase cells by observing and searching through a large number of potential metaphase cells depicted on all slides prepared for a patient. As a result, visually searching for and diagnosis of these analyzable metaphase cells is a labor-intensive and time-consuming process (16). In addition, this procedure introduces a large inter- and intra-reader variability, which may cause substantial error in the evaluation or prediction of prognosis for the patients' treatment.

In order to improve detection efficiency and sensitivity, development of automated chromosome detection has attracted great research interests since 1980s. Several computer schemes have been developed in an attempt to identify and analyze chromosomes (17-19). Most of them are semi-automatic schemes and the operator intervention is often required. Using these interactive metaphase finding and karyotyping systems, genetic laboratory technologists visually verify the identified metaphase cells, select analyzable cells, and then use the mouse, light pens, and other methods to segment metaphase clusters (20-23), and correct separations between touching and overlapping chromosomes (24). Several commercial systems including Magiscan (Joyce Loebl, Gateshead UK), Cytoscan (Image Recognition Systems, Warrington, UK), AKS-2 systems (Amoco Technology Inc. Naperville, IL), and Genetiscan (Perceptive Scientific Instruments Inc., League City, TX), have been used in some genetic laboratories to assist automatic metaphase chromosome finding and karyotyping task (25). Due to the continuous research efforts, several new techniques have been recently investigated and developed to improve accuracy in identification of metaphase chromosomes. These included a knowledgebased chromosome contour searching method (26), a novel recursive algorithm (27), minimum entropy segmentation (28), and a rule-based approach to realize fully automatic chromosome segmentation (29). The most of these methods are often computing intensive. Some algorithms take several hours to segment one image (28). In addition, the robustness of these methods has not been adequately investigated.

To improve the efficiency of detecting analyzable metaphase chromosome cells and to predict prognosis of ALL, we developed and tested a new computer-aided method and scheme to automatically detect metaphase chromosomes depicted on a microscopic digital image, count the total number of individual chromosomes depicted on one metaphase cell, and compute the DNA index. The detailed description of the scheme, along with the experimental results when applying the scheme to 60 images acquired from three pediatric patients at different categories of ALL prognosis, is presented here.

\section{Materials and Methods}

We select 60 chromosome images from three pediatric patients with ALL from a genetics laboratory at the University of Oklahoma Health Science Center. Those chromosome images are captured by an optical microscope with objective lenses of 100X magnification power, the binocular eyepiece 


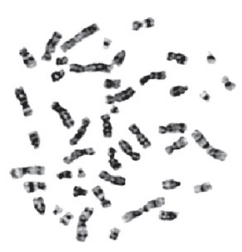

a

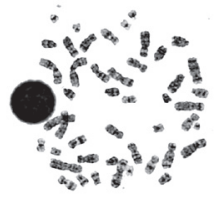

e

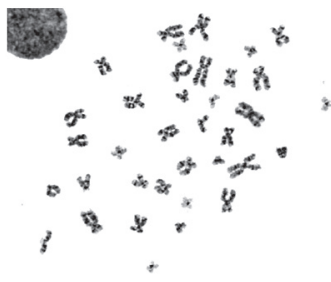

b

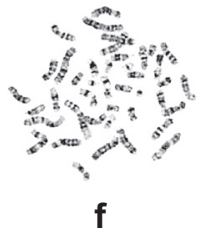

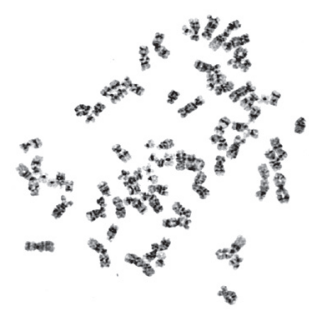

C

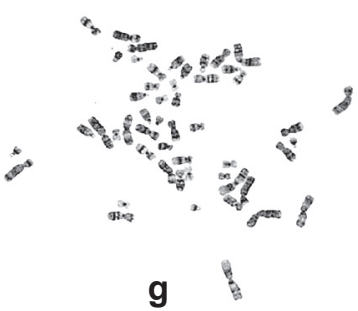

Figure 1: Analyzable metaphase chromosome images from three groups. (a) (e) Category I: The number of chromosomes is equal to 46; (b) (f) Category II: The number of chromosomes is less than 46; and (c) (g) Category III: The number of chromosomes is more than 46 . lenses of 10X magnification, and a digital camera. The physical pixel size of those images is $0.2 \mu \mathrm{m} \times$ $0.2 \mu \mathrm{m}$. Those chromosome images have an average size of 768 $\times 576$ pixels and cells are stained with Giemsa. The 20 chromosome images were acquired from each child patient diagnosed with three different categories of ALL (peudodiploid, hypodiploid, and hyperdiploid). Figure 1 demonstrates six examples from these three categories. An experienced cytogeneticist counted the number of the chromosomes in each of these 60 images. The results were saved in a "truth" file.

Figure 2 describes the detailed flow chart of this computer-aided detection and analysis scheme. The scheme includes the following major components.

\section{Adjustable Threshold}

The first step in this method is to segment the suspicious chromosomes through an initial threshold and then in the following iterative steps, re-segment the actual chromosomes using a variable (adaptively adjusted) threshold

Figure 2: A flow chart of a computer-aided scheme for detecting chromosomes and counting the number of chromosomes depicted on one image.

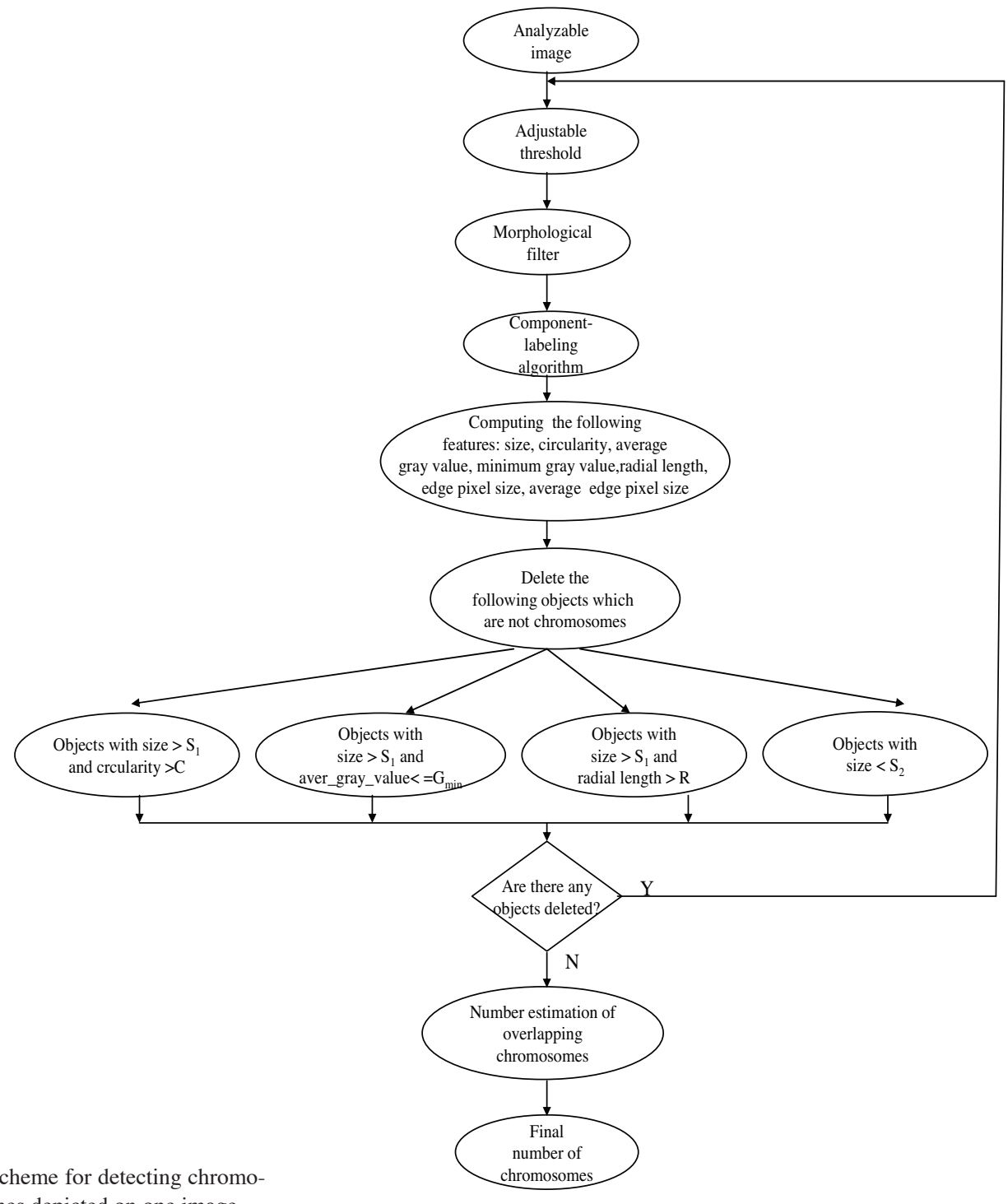


to further delete other inter-phase cells, stain debris, and background noise. Because the value of the threshold is changed in re-segment iterative steps, this threshold is adjustable. In the initial segment, since the objects have much lower gray values than the background, the initial threshold should be large. In the re-segment steps, we should choose an appropriate threshold according to the slope of the gray value histogram of the image.

\section{Morphological Filter}

In the second step, we utilize a morphological filter to remove small objects and holes linked to initially detected chromosomes. Specifically, a $3 \times 3$ open filter was used to get rid of the background noise and small objects such as stain debris. Moreover, this open filter can also disconnect some of the partially touching chromosomes.

\section{Component Labeling Algorithm}

In the third step, with the binary "noise free" image generated in the second step, we count the number of objects based on a 4-connectivity component-labeling algorithm (30). The labeling algorithm includes the following steps.

I. Scan the image from top to bottom and left to right (pixel-by-pixel)

II. If the pixel $p$ is logic one, examine the upper and the left of $p$, the labeling of $p$ occurs as follows:

a. If the upper and left neighbors are not logic one (object), then we assign a new label to $p$, and record this label in the equivalence table;

b. If only one neighbor has a label, assign its label to $p$;

c. If both of the neighbors have the same label, then copy it;

d. If both of the neighbors have different labels, assign the smallest one of the labels to $p$ and make a note of the equivalences;

III. After completing the scan, the equivalent label pairs are sorted into equivalence classes and a unique label is assigned to each class.

IV. Scan the image again, during which each label is replaced by the label assigned to its equivalence classes.

\section{Feature Selection}

After the computer scheme detected all the separated objects through a component-labeling algorithm, it computed a set of seven features from each object in attempt to better classify between chromosomes and other un-related objects (noise) in the fourth step. A list of features is described as follows:
I. The size of each labeled object: It is gained by counting the number of pixels contained in the region $\left(N_{m}\right)$;

II. The circularity of each labeled object: Based on the area size of the object $\left(N_{m}\right)$, the method defines an equivalent circle originating at the gravity center of the object, and computes the number of pixels that are located inside the object contour and the circle $\left(N_{C}\right)$, which has the same size as the labeled object. The circularity is defined as $C_{i}=\frac{N_{C}}{N_{m}}$, the ratio of the region pixels covered by the circle to the total pixels inside the labeled region;

III. The average gray value of each object: It is computed as an average digital value of pixels

$$
\left(G_{\text {ave }}=\frac{1}{N_{m}} \sum_{i=1}^{N_{m}} G_{i}\right) ;
$$

IV. The minimum average gray value of all objects: It is obtained by $G_{\min }=\operatorname{Min}\left(\sum_{i=1}^{N_{m}} G_{\text {aver_i }}\right)$;

V. The distance of each object to the cell center (radial length): It is defined as the distance between the gravity center $\left(x_{c}, y_{c}\right)$ of total labeled regions in the image (center of a cell) and the center of one individual region $\left(x_{k}, y_{k}\right)$. It is computed as

$$
D_{k}=\sqrt{\left.x_{c}-x_{k}\right)^{2}+\left(y_{c}-y_{k}\right)^{2}} .
$$

VI. The perimeter of each object: this feature is computed by counting the number of edge pixels in the object $\left(P_{i}\right)$;

VII. The average perimeter of all objects is defined as $P_{\text {ave }}=\frac{1}{N} \sum_{i=1}^{N} P_{i}(\mathrm{~N}$ is the number of all objects in an identified cell).

\section{Deleting Non-chromosome Objects}

Based on the seven features, a knowledge-based classifier (a set of rules) was applied to delete those non-chromosome objects, which include inter-phase cells, stain debris, and other types of artificial background noise. These empirical rules were decided based on our visual observations and discussion with the cytogeneticists. For example, the inter-phase cells are typically large, circular, and dark, but sometimes their shape are also irregular, and their gray value are very low. In general, our classifier included following four rules to identify and delete inter-phase cells and stain debris (as well as other small irregular objects):

I. Regular and round inter-phase cells: the size of an object is larger than $S_{1}$ and the circularity is larger than $\mathrm{C}\left(\mathrm{S}_{1}\right.$ is larger than six times of the average size of objects in the image, and $\mathrm{C}$ is equal to 0.90 ); 
II. Irregular inter-phase cells I: the size of an object is larger than $S_{1}$ and the gray value of this object is smaller than or equal to the minimum average gray value of all objects in the cell;

III. Irregular inter-phase cells II: the size of an object is larger than $\mathrm{S}_{1}$ and it is far away from the gravity center in the chromosome image (a cell), and its radial length is larger than $R$ [ $R$ equals to (maximum radial length - standard deviation of radial length of all objects)];

IV. Stain debris or other small irregular objects (noise): the size of an object is larger than $\mathrm{S}_{2}$, which is equal to 90 pixels or $1 / 10$ of the average size of all objects.

To achieve the optimal results, the scheme iterates above steps to detect and delete as many non-chromosome objects as possible. As long as there are any non-chromosome objects detected in the iteration (using above four classification criteria), the scheme sets flag $=$ false and automatically performs a new iteration. The scheme stops the iteration, if there is no single non-chromosome object detected (when the scheme sets flag $=$ true). Figure 3 gives a detailed example result which successfully deletes those objects that are not metaphase chromosomes. The performance of these classification rules has been tested in our previous study (31) using a database of 100 images including both analyzable and unanalyzable metaphase cells.

\section{Counting the Number of Chromosomes}

Since a number of chromosomes can be overlapped (as shown in Figure 3), the number of chromosomes counted in one image after above image processing (including the use of a labeling algorithm) often under-estimates the actual number of chromosomes. In order to detect these overlapped chromosomes and count them correctly, in the last stage of our computer scheme, we estimate and count the overlapping chromosomes. A number of techniques have previously been developed and reported by other research groups to segment the overlapped chromosomes (26-29). The estimated accuracy of these algorithms is typically in the range between $70 \%$ and $80 \%$. The biggest obstacles of these methods are their computational complexity and inefficiency.

Because in this study our goal is to count the number of chromosomes in one metaphase chromosome image, as long as the computer scheme can detect how many chromosomes are overlapped, the scheme can adjust (correct) the counted number of total chromosomes without physical segmentation (separation) of overlapped chromosomes. Our hypotheses are that: (i) by avoiding the complex and often unreliable process of decomposing (or separating) the overlapped chromosomes, the computer scheme can be much more simple and efficient; (ii) based on the ratio of the area size of an overlapped object to the average size of other objects, the scheme can estimate how many chromosomes are included in this overlapped object, and then add that number to the total number of chromosomes in one test metaphase image. For this purpose, we used the following criteria to identify or determine whether one or more chromosomes are overlapping. The criterion is when the area size of an object is larger than two times the average area size of all objects and the perimeter of this object is also larger than two times the average perimeter of all objects, the computer scheme will consider this labeled object involves overlapped chromosomes and correspondingly increase the counted number of chromosomes in this test image.

\section{Computing DNA Index}

After completing the detection and count process for all testing slices (the microscopic images depicting analyzable metaphase chromosomes) acquired in one case (one patient), the scheme computes the DNA index of the case, $D I=\frac{N_{\text {ave }}}{N_{\text {norm }}}$. where $N_{\text {ave }}$ is the average number of chromosomes detected and counted in all testing images of a pediatric patient and $N_{\text {norm }}=46$ (since a normal metaphase cell should include 46 chromosomes). This index can be used to correlate or predict the prognosis of ALL.

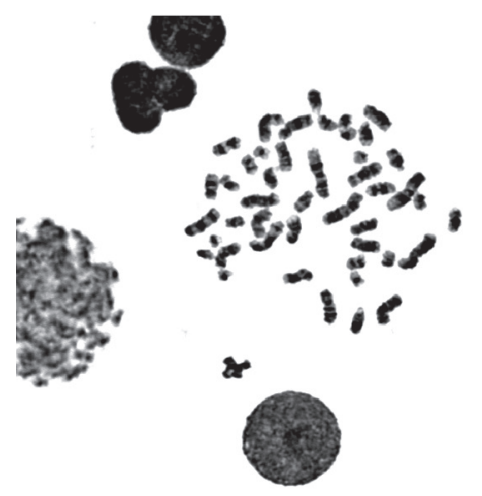

(a)

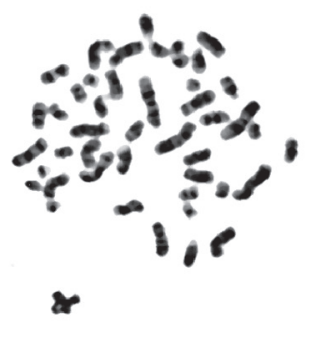

Figure 3: One example of eliminating the nonchromosome objects. (a) Original image and (b) processed image. 
Table I

The Prognosis analysis result for three groups.

\begin{tabular}{|c|c|c|c|c|c|c|c|c|c|c|}
\hline \multirow{2}{*}{$\begin{array}{c}\text { Testing } \\
\text { Case }\end{array}$} & \multicolumn{5}{|c|}{ Numbers of images by chromosome counts } & \multirow{2}{*}{ Average } & \multirow{2}{*}{$\begin{array}{l}\text { Range of } \\
\text { Numbers }\end{array}$} & \multirow{2}{*}{ DI } & \multirow{2}{*}{ Category } & \multirow{2}{*}{$\begin{array}{l}\text { Prognosis } \\
\text { Evaluation }\end{array}$} \\
\hline & $<45$ & 45 & 46 & 47 & $>47$ & & & & & \\
\hline I & 1 & 5 & 12 & 2 & 0 & 45.8 & $44 \sim 47$ & 0.99 & Pseudodiploid & Poor \\
\hline II & 19 & 1 & 0 & 0 & 0 & 41.4 & $36 \sim 45$ & 0.90 & Hypodiploid & Poor \\
\hline III & 0 & 0 & 0 & 0 & 20 & 56.5 & $48-65$ & 1.22 & Hyperdiploid & Good \\
\hline
\end{tabular}

\section{Results}

Using visual assessment, we found that the number of chromosomes counted by the computer scheme for each of identified metaphase cells is similar to the corresponding number recorded in the "truth" file (with difference $\leq \pm 2$ chromosomes for a metaphase cell). Table I summarizes the statistical data and analytic results of the prognosis for three test cases (pediatric patients diagnosed with ALL), while Figure 4 demonstrates the plots of the distribution of the number of chromosomes in these three testing cases. The average number of chromosomes from patient I is 45.8 , which is approximately 46, and DNA index (DI) is $0.99(45.8 / 46)$. Based on the average number of the chromosomes in one metaphase cell and DI of the patient, the scheme classified this case as a pseudodiploid category ALL. The number of chromosomes counted in each of 20 images from case II is less than 46 . The average number is 41.4 with DI of 0.90 (41.4/46). Therefore, case II was classified to be a hypodiploid category ALL. All of the 20 images from case III have more than 46 chromosomes. The average number of chromosomes in case III is 56.5 and DI is $1.22(56.5 / 46)$ (between 1.16 and 1.6). According to the number of chromosomes and DI, the computer scheme classified case III as a hyperdiploid category ALL.

\section{Discussion}

The identification of genetic features can help clinicians predict and evaluate cancer prognosis, as well as select optimal treatment procedures for the patients with the appropriate levels of chemical and/or radiation therapies. In this preliminary study, we described a computerized method (scheme) to automatically detect metaphase chromosomes depicted on a digitally acquired microscopic image and count the total number of detected chromosomes in a metaphase cell. One of the main purposes of developing a computerized method or scheme is to replace the tedious and inefficient process commonly used in genetic diagnostic laboratories to perform chromosome image analysis (including visually searching for and identifying analyzable metaphase chromosomes as well as counting individual chromosomes and calculating DNA index or other features). Because the technologists in the genetic laboratory typically randomly select 20 to 30 metaphase chromosome cells from a large number of potentially analyzable cells, errors and inter-reader variations can occur in the diagnosis of subtle cases and the evaluation of

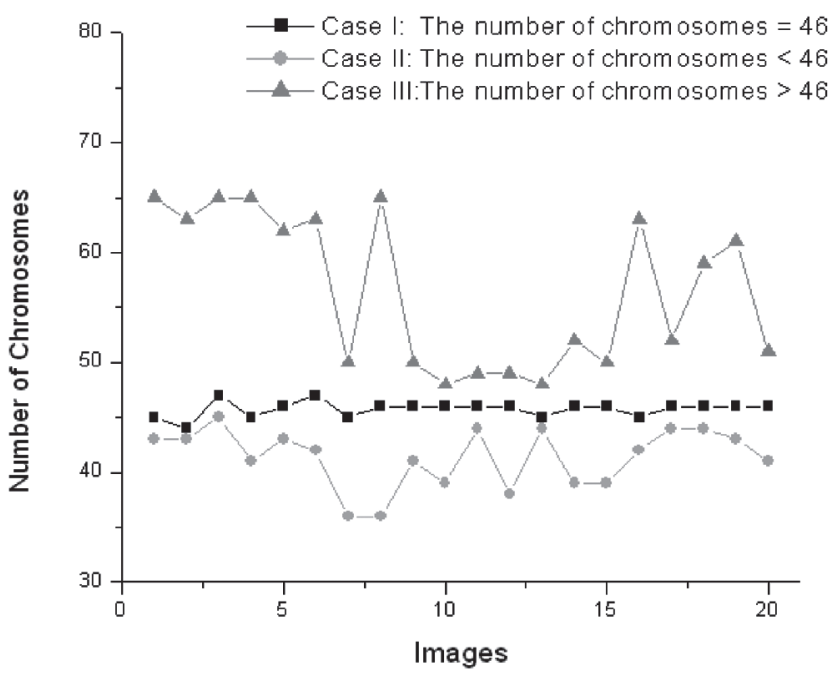

Figure 4: Distribution of the number of chromosomes in three testing cases.

treatment efficacy. In our laboratory we have been working to develop a comprehensive computer-aided detection and diagnosis scheme for the diagnosis of chromosome images. The overall scheme was designed to include a number of independent modules. The first developed module is designed to automatically identify analyzable metaphase chromosomes and delete inter-phase cells and stain debris (31). This study focused on the development of the second module. Due to the difficulty in segmentation of overlapped chromosomes, the schemes previously developed (24) have not been used to automatically count the number of chromosomes in each identified metaphase cell. One unique characteristic of our scheme is that it can count the number of chromosomes without segmenting overlapped chromosomes. In addition, by computing the average number of chromosomes depicted on a large number of metaphase cells acquired from one patient, the scheme may minimize the negative impact due to the sample slide preparation errors that may cause chromosome gain or loss and improve diagnostic accuracy.

This study demonstrates that our computer-aided scheme can reliably detect individual chromosomes depicted on a noisy image and count the total number of chromosomes involved in a metaphase cell after compensating the effect of chromosome overlapping. The advantage of using this computer scheme is that it cannot only replace the tedious (inefficient) process of visual searching and counting, but also help clini- 
cians report more consistently diagnostic results. In this study, our scheme predicted that patient III had a good prognosis and the outcome of the treatment was usually good. On the other hand, patients I and II had a poor prognosis and, therefore, a more aggressive treatment should be recommended. These conclusions are highly correlated with those analyzed and recommended by the clinicians in our genetic laboratory.

This preliminary study has several limitations. First, if some of the analyzable metaphase chromosomes are overlapped by the irregular inter-phase cells, those chromosomes are likely to be considered a part of inter-phase cells and be eliminated. For example, this is the primary reason that the number of chromosomes in some metaphase cells (images) from case I is slightly different from the number of 46 . In our future research, we need to test and optimize the computing algorithms used in the scheme with more chromosome images acquired from more patients within different age groups and different genders. We will investigate and search for more effective methods to recognize the overlapped chromosomes and optimally compensate the counting results. Second, the size of the testing dataset used in this study is very limited. We are currently acquiring more cases to increase the size and diversity of our dataset. The robustness of the scheme will be evaluated in our future studies.

\section{Acknowledgement}

This research was supported in part by grants from the National Institute of Health (CA104773 and EB002604). The authors would like to acknowledge the support of Charles and Jean Smith Chair endowment fund as well.

\section{References}

1. A. M. Friedmann, H. J. Weinstein. The Role of Prognostic Features in the Treatment of Childhood Acute Lymphoblastic Leukemia. The Oncologist 5, 321-328 (2000).

2. V. Conter, C. Rizzari, A. Sala, R. Chiesea, M. Citterio, A. Biondi. Acute Lymphoblastic Leukemia. Orphanet Encyclopedia. December 2004. http://www.orpha.net/data/patho/GB/uk-ALL.pdf

3. E. Olah, E. Balogh, P. Kajtar, L. Pajor, Z. Jakab, C. Kiss. Diagnostic and Prognostic Significance of Chromosome Abnormalities in Childhood Acute Lymphoblastic Leukemia. Ann. NY Acad. Sci. 824, 8-27 (1997).

4. W. L. Carroll, D. Bhojwani, D. J. Min, E. Raetz, M. Relling, S. Davies, J. R. Downing, C. L. Willman, J. C. Reed. Pediatric Acute Lymphoblastic Leukemia. Hematology 2003, 102-31 Review.

5. G. Al-Harbi, H. El-Solh, A. Al-Nasser, S. Khalil, et al. DNA Index in Childhood Acute Lymphoblastic Leukemia: Correlation with Other Prognostic Factors, Pediatric Oncology. 2000 ASCO Annual Meeting.

6. V. M. Whitehead, M. J. Vuchich, S. J. Lauer, D. Mahoney, A. J. Carroll, Shuster J. J., D. W. Esseltine, C. Payment, A. T. Look, J. Akabutu, et al. Accumulation of High Levels of Methotrexate Polyglutamates in Lymphoblasts From Children with Hyperdiploid (Greater than 50 Chromosomes) B-lineage Acute Lymphoblastic Leukemia: A Pediatric Oncology Group Study. Blood 80, 1316-1323 (1992).

7. D. L. Williams, A. Tsiatis, G. M. Brodeur, A. T. Look, S. L. Melvin, W. P. Bowman, D. K. Kalwinsky, G. Rivera, G. V. Dahl. Prognostic
Importance of Chromosome Number in 136 Untreated Children with Acute Lymphoblastic Leukemia. Blood 60, 864-871 (1982).

8. L. M. Secker-Walker, G. J. Swansbury, R. M. Hardisty, S. E. Sallan, O. M. Carson, M. Sakurai, S. D. Lawler. Cytogenetics of Acute Lymphoblastic Leukemia in Children as a Factor in the Prediction of Long-term Survival. Br. J. Haemato. 52, 389-399 (1982).

9. C. D. Bloomfield, L. M. Secker-Walker, A. I. Goldman, Van Den, H. Berghe, A. de la Chapelle, T. Ruutu, G. Alimena, O. M. Carson, H. M. Golomb, J. D. Rowley, Y. Kaneko, J. Whang-Peng, E. Prigogina, P. Philip, A. A. Sandberg, S. D. Lawler, F. Mitelman. Six-year Follow-up of the Significance of Karyotype in Acute Lymphoblastic Leukemia. Cancer Genet. Cytogenet. 40, 171-185 (1989).

10. A. T. Look, S. L. Melvin, S. L. Williams, G. M. Brodeur, G. V. Dahl, D. K. Kalwinsky, S. B. Murphy, A. M. Mauer. Aneuploidy and Percentage of S-phase Cells Determined by Flow Cytometry Correlate with Cell Phenotype in Childhood Acute Leukemia. Blood 60, 959967 (1982)

11. A. T. Look, P. K. Roberson, D. L. Williams, G. Rivera, W. P. Bowman, C. H. Pui, J. Ochs, M. Abromowitch, D. Kalwinsky, G. V. Dahl, S. George, S. B. Murphy. Prognostic Importance of Blast Cell DNA Content in Childhood Acute Lymphoblastic Leukemia. Blood 65 , 1079-1086 (1985).

12. L. A. Smets, J. Homan-Blok, A. Hart, G. de Vaan, H. Behrendt, K. Hahlen, F. J. de Waal. Prognostic Implication of Hyperdiploidy as Based on DNA Flow Cytometric Measurement in Childhood Acute Lymphocytic Leukemia-A Multicenter Study. Leukemia 1, 163 166 (1987)

13. R. Trueworthy, J. Shuster, T. Look, W. Crist, M. Borowitz, A. Carroll, L. Frankel, M. Harris, H. Wagner, M. Haggard, A. Mosijczuk, J. Pullen, P. Steuber, V. Land. Ploidy of Lymphoblasts is the Strongest Predictor of Treatment Outcome in B-progenitor Cell Acute Lymphoblastic Leukemia of Childhood: A Pediatric Oncology Group Study. Journal of Clinical Oncology 10, 606-613 (1992).

14. C. H. Pui, W. M. Crist. High Risk Lymphoblastic Leukemia in Children: Prognostic Factors and Management. Blood Rev. 1, 25-33 (1987).

15. C. H. Pui, D. L. Williams, S. C. Raimondi, G. K. Rivera, A. T. Look, R. K. Dodge, S. L. George, F. G. Behm, W. M. Crist, S. B. Murphy. Hypodiploidy is Associated with a Poor Prognosis in Childhood Acute Lymphoblastic Leukemia. Blood 70, 247-253 (1987).

16. X. Wang, B. Zheng, M. Wood, S. Li, W. Chen, H. Liu. Development and Evaluation of Automated Systems for Detection and Classification of Banded Chromosomes: Current Status and Future Perspectives. J. Phys. D: Appl. Phys. 38, 2536-2542 (2005).

17. J. Piper, E. Granum, D. Rutovitz, H. Ruttledge. Automation of Chromosome Analysis. Signal Processing 2, 203-221 (1980).

18. J. Liang, J. Piper, J. Y. Tang. Erosion and Dilation of Binary Images by Arbitrary Structuring Elements Using Interval Coding. Pattern Recognition Letters 9, 201-209 (1989).

19. A. M. Vossepoel. Separation of Touching Chromosomes Chapter in Automation of Cytogenetics, pp. 205-216. Eds., C. Lundsteen, J. Piper. Springer-Verlag, Berlin (1989).

20. J. Liang. Intelligent Splitting in the Chromosome Domain. Pattern Recognition 22, 519-532 (1989).

21. J. Graham. Resolution of Composites In Interactive Karyotyping, Chapter in Automation of Cytogenetics , pp. 191-203. Eds., C. Lundsteen, J. Piper. Springer-Verlag, Berlin (1989).

22. A. M. Vossepoel. Separation of Touching Chromosomes, In Automation of Cytogenetics, pp. 205-216. Eds., C. Lundsteen, J. Piper. Springer-Verlag, Berlin (1989).

23. Q. Wu, J. Snellings, L. Amory, P. Suetens, A. Oosterlinck. Modelbased Contour Analysis in a Chromosome Segmentation System, In Automation of Cytogenetics, pp. 217-229. Eds., C. Lundsteen, J. Piper. Springer-Verlag, Berlin (1989).

24. A. Carothers, J. Piper. Computer-aided Classification of Human Chromosomes: A Review. Stat. Comput. 4, 161-171 (1994). 
25. J. Graham, J. Piper. Automatic Karyotype Analysis, Chapter In Methods in Molecular Biology: Chromosome Analysis Protocols, Vol. 29, pp. 141-186. Ed., J. R. Gosden. Totowa, Humana Press Inc. (1994).

26. G. Agam, I. Dinstein. Geometric Separation of Partially Overlapping Nonrigid Objects Applied to Automatic Chromosome Classification. IEEE Trans Pattern Analysis and Machine Intelligence 19, 12121222 (1997).

27. M. Popescu, P. Gader, J. Keller, C. Klein, et al. Automatic Karyotyping of Metaphase Cells with Overlapping Chromosomes. Comput. Biol. Med. 29, 61-82 (1999).

28. W. Schwartzkopf, B. L. Evans, A. C. Bovik. Minimum Entropy Segmentation Applied to Multi-spectral Chromosome Images. 2001 International Conference on Image Processing 2, 865-868 (2001).
29. J. Liang. Fully Automatic Chromosome Segmentation. Cytometry 17, 196-208 (1994)

30. R. Jain, R. Kasturi, B. G. Schunck. Machine Vision. McGraw-Hill, Inc., New York, NY (1995).

31. X. Wang, S. Li, H. Liu, M. Wood, W. Chen, B. Zheng. A Computerized Method to Segment and Classify Metaphase Chromosomes. Submitted to Compt. Biol. Med. (2005).

32. B. Lerner. Toward a Completely Automatic Neural-network-based Human Chromosome Analysis. IEEE Trans Systems, Man, and Cybernetics - Part B: Cybernetics 28, 544-552 (1998).

Received: September 23, 2005; Revised: February 22, 2006; Accepted: March 8, 2006 